\title{
Assessing recent time-dependent double-hybrid density functionals on doublet-doublet excitations
}

\author{
Joshua Van Dijk, ${ }^{\dagger}$ Marcos Casanova-Páez, ${ }^{\dagger}, \ddagger$ and Lars Goerigk ${ }^{*, \dagger}$ \\ $\dagger$ School of Chemistry, The University of Melbourne, Victoria 3010, Australia \\ $\ddagger$ Max-Planck-Institut für Kohlenforschung, Kaiser-Wilhelm Platz 1, 45470 Mülheim an der \\ Ruhr, Germany \\ E-mail: lars.goerigk@unimelb.edu.au
}

Phone: +61383446784

\section{Abstract}

This work is the first thorough investigation of time-dependent double hybrid density functionals (DHDFs) for the calculation of doubletdoublet excitation energies. It sheds light on the current state-of-the-art techniques in the field and clarifies if there is still room for future improvements. Overall, 29 hybrid functionals and DHDFs are investigated. We separately analyze the individual impacts of the Tamm-Dancoff approximation (TDA), range separation, and spin-component/opposite scaling (SCS/SOS) on 45 doublet-doublet excitations in 23 radicals before concluding with an overarching analysis that includes and excludes challenging excitations with double-excitation or multi-reference character. Our results show again that so-called 'non-empirical' DHDFs are outperformed by semi-empirical ones. While the best assessed functionals are DHDFs, some of the worst are also DHDFs and outperformed by all assessed hybrids. SCS/SOS is particularly beneficial for range-separated DHDFs. Spin-scaled, range-separated DHDFs paired with the TDA belong to the best tested methods here and we particularly highlight SCS- $\omega$ B2GP-PLYP, SOS- $\omega$ B2PLYP, and SOS$\omega$ B2GP-PLYP, SOS- $\omega$ B88PP86, SOS-RSXQIDH, and SOS- $\omega$ PBEPP86. When comparing our functional rankings with previous stud- ies on singlet-singlet and singlet-triplet excitations, we recommend TDA-SOS- $\omega \mathrm{B} 88 \mathrm{PP} 86$ and TDA-SOS- $\omega$ PBEPP86 as robust methods for excitation energies in general until further improvements have been achieved that surpass the chemical accuracy threshold for challenging excitations without increasing the computational effort.

\section{Introduction}

Density Functional Theory ${ }^{112}$ (DFT) is the most popular methodology for computational chemistry calculations, in large part due to its efficiency compared to methods based entirely on wave function theory (WFT). Despite DFT being exact in principle, the functionals that describe electron correlation and exchange are not known and must be approximated. The success of DFT is therefore highly dependent on the selection of the density functional approximation (DFA). The number of available DFAs continues to grow steadily, with each new DFA attempting to more accurately describe challenging quantum-mechanical effects without significantly increasing computational cost. Initial DFT methods, based on the HohenbergKohn theorems ${ }^{1}$ and the Kohn-Sham equations, ${ }^{2]}$ were only applicable to ground-state problems. In 1984, Runge and Gross devel- 
oped equivalent theorems for excited-state calculations, ${ }^{3}$ paving the way for time-dependent DFT (TD-DFT). As TD-DFT requires an initial ground-state DFT calculation as input, its success is also dependent on the underlying DFA. In TD-DFT calculations, matrices for both single-particle excitations and deexcitations are used. However, computational cost can be decreased by using only the excitation matrix. This simplification is known as the Tamm-Dancoff approximation (TDA) leading to TDA-DFT applications. ${ }^{4}$

When considering DFT's metaphorical 'Jacob's Ladder', 5 the description of electronic excitations with linear-response TD-DFT only becomes reasonable by using good representatives from at least the fourth rung of the ladder, ${ }^{6} 8$ namely hybrid DFAs, which combine semi-local exchange and correlation from DFT with non-local Fock exchange originally derived for WFT. Even better results for excitation energies are obtained when going to the fifth rung, namely double-hybrid density functionals (DHDFs) ${ }^{910}$ which improve the description of electron correlation by adding information from unoccupied (virtual) molecular orbitals in the form of a second-order perturbative component. Indeed, TD(A)-DHDFs following an approach suggested by Grimme and Neese in $2007^{11}$ are known to be the most accurate DFAs for valence excitation energy calculations and absorption spectra in organic molecules, 6,8$] 10,19$ in some cases rivalling standard WFT methods. $7114 \mid 17+21$

In the past, several attempts have been made to improve upon the initial $\mathrm{TD}(\mathrm{A})$ DHDFs. Most notably those were the introduction of spin-component and spin-opposite scaling $22 \sqrt{25}$ (SCS/SOS) to the perturbative part by Schwabe and Goerigk in 2017, 17 and the introduction of range-separation ${ }^{26}+29$ to nonSCS/SOS TD-(A)DHDFs by Casanova-Páez, Dardis, and Goerigk in 2019. 18 The latter improvement was an important step forward because prior to $2019 \mathrm{TD}(\mathrm{A})$-DHDFs following the Grimme-Neese idea had had the same incorrect decay of the exchange potential and electron density as other global DFAs. $.7 / 18|19| 30 \mid 33$ This incorrect decay had caused global DHDFs to inadequately describe long-range transitions, in particular charge-transfer (CT) excitations. One popular attempt to combat this issue is range-separation, also known as long-range correction, 26 29 which has been successfully applied to hybrid DFAs. The aforementioned work in 2019 follows the same spirit and introduced the first range-separated TD-DHDFs optimized for excitation energies called $\omega$ B2PLYP and $\omega$ B2GP-PLYP. ${ }^{18}$ They seamlessly connect hybrid-DFT exchange in the short-range regime of the electron-electron distance with $100 \%$ Fock exchange in the long range. Establishing $100 \%$ Fock exchange in the long range has been the underlying principle in most rangeseparated DFAs in the past, with the CAMB3LYP hybrid being a notable exception. ${ }^{28}$ The strongly improved performance of $\omega$ B2PLYP and $\omega$ B2GP-PLYP has been demonstrated for singlet-singlet and singlet-triplet transitions, including for some infamously challenging localvalence, Rydberg, and CT excitations, 18 [19/30 such as polycyclic aromatic hydrocarbons. 18

Further improvements of global and rangeseparated - in the exchange part-TD(A)DHDFs have been achieved by our group in 2021 by revisiting the impact of SCS and SOS techniques. $21^{21}$ In SCS/SOS, the perturbative component of the electron correction energy is split into energies of electron pairs of same and opposite spin and those components are individually scaled. For instance, in the original SCS-MP2 from 2003 this prevented the overestimation of same-spin correlation. ${ }^{22}$ By ignoring the same-spin part, SOS-based methods can achieve an improved formal scaling behavior, which makes them particularly valuable for larger systems. $\stackrel{23}{2}$ The superior performance of some of the fourteen developed SCS/SOSbased TD(A)-DHDFs has been demonstrated for singlet-singlet and singlet-triplet excitations with the best methods outperforming our original 2019 approaches, as well as some alternative DHDF definitions published in 2021, $34 \sqrt[35]{3}$ wich also employ range-separation in the perturbative part.21

Nearly all benchmark studies exploring TD(A)-DHDFs dealt with closed-shell systems and assessments of open-shell systems have 


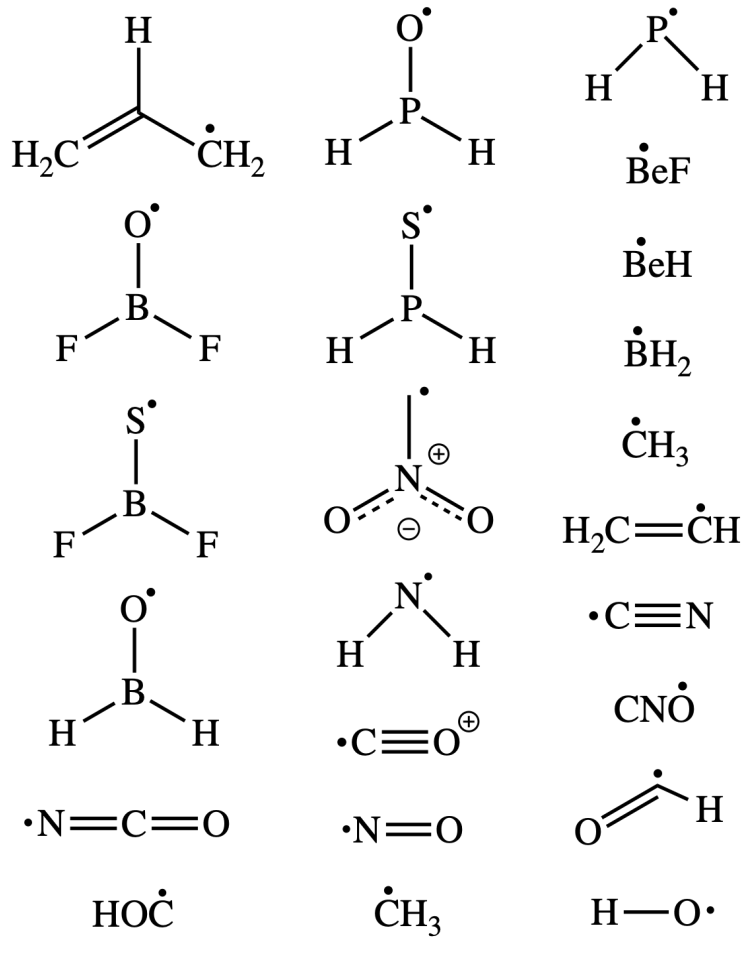

Figure 1: A set of 23 radicals published in Ref. 38 .

been rare and very limited in scope. In 2007, Grimme and Neese assessed TDA-B2PLYP on 11 small radicals. 11 The only other two examples in the literature are the limited study of six open-shell atoms ${ }^{36}$ and seven molecular openshell systems ${ }^{37}$ with four global DHDFs. To our knowledge, no systematic study of openshell excitations has been conducted that also involves the latest developments in this area. Herein, we intend to close this knowledge gap. Our study will be conducted on a set of 45 doublet-doublet excitations in 23 molecular radicals initially presented and analyzed with WFT methods by Loos, Jacquemin and coworkers (Fig. 1). ${ }^{38}$ In particular, we will compare TD(A)-DHDFs with hybrid functionals and separately address the impact of the TDA, range-separation and SCS/SOS on the excitation energies.

This is the most thorough study of DHDFs on open-shell systems and it will provide insights on the current state-of-the-art in the field of DHDFT for excitation energies, which will inform us of whether they perform as well as for closed-shell systems or whether there is the necessity for further developments.

\section{Theoretical background}

According to Grimme, a DHDF consists of two components: a hybrid component and an additional second-order perturbative correlation term. ${ }^{9}$ Herein, we only discuss DHDFs that include range-separation solely in the exchange part, but range-separation has also been suggested in the correlation portion. $\frac{3435}{35}$ Details on time-dependent DHDFs with and without SCS/SOS have been extensively provided in the literature, ${ }^{8 / 17 \mid 21}$ here we only discuss the basics.

Vertical excitation energies for real orbitals are computed by solving the random-phase approximation (RPA) problem: $\underline{39}$

$$
\left(\begin{array}{cc}
\mathbf{A} & \mathbf{B} \\
\mathbf{B} & \mathbf{A}
\end{array}\right)\left(\begin{array}{c}
\mathbf{X} \\
\mathbf{Y}
\end{array}\right)=\Delta E_{\mathrm{TD}-\mathrm{DFT}}\left(\begin{array}{cc}
\mathbf{1} & \mathbf{0} \\
\mathbf{0} & -\mathbf{1}
\end{array}\right)\left(\begin{array}{c}
\mathbf{X} \\
\mathbf{Y}
\end{array}\right),
$$

where $\Delta E_{\text {TD-DFT }}$ is the vertical excitation energy and $\mathbf{X}$ and $\mathbf{Y}$ are the eigenvectors for single-particle excitations and de-excitations, respectively. $\mathbf{A}$ and $\mathbf{B}$ are matrices containing information on these excitations and deexcitations. By removing the $\mathbf{B}$ matrix from Eq. 1, a simplified equation-the TammDancoff approximation $\underline{4}$ is obtained:

$$
\mathbf{A X}=\Delta E_{\text {TDA-DFT }} \mathbf{X}
$$

According to Grimme and Neese, vertical excitation energies at the double-hybrid level are obtained by first solving the TD(A)-DFT eigenvalue problem with only the hybrid portion of the DHDF ( $\Delta E_{\mathrm{TD}(\mathrm{A}) \text {-hybrid }}$ in Eq. 3), and then adding Head-Gordon and co-workers' CIS(D) perturbative correction $\left(\Delta_{\mathrm{CIS}(\mathrm{D})}\right): \underline{40}$

$$
\Delta E_{\mathrm{TD}(\mathrm{A})-\mathrm{DHDF}}=\Delta E_{\mathrm{TD}(\mathrm{A}) \text {-hybrid }}+a_{C} \Delta_{\mathrm{CIS}(\mathrm{D})} .
$$

The parameter $a_{C}$ in Eq. 3 scales the CIS(D) contribution akin to ground-state DHDF calculations. 11

According to Schwabe and Goerigk, ${ }^{17} \mathrm{TD}(\mathrm{A})$ SCS/SOS-DHDF excitation energies are obtained by adding a SCS/SOS-CIS(D) $)^{24}$ correction $\left(\Delta_{\mathrm{SCS} / \text { Sos-CiS(D) }}\right)$ to the $\mathrm{TD}(\mathrm{A})$-hybrid energy: 
$\Delta E_{\mathrm{TD}(\mathrm{A}) \text {-SCS} / \mathrm{SOS}-\mathrm{DHDF}}=\Delta E_{\mathrm{TD}(\mathrm{A}) \text {-hybrid }}+\Delta_{\mathrm{SCS} / \mathrm{SOS}-\mathrm{CIS}(\mathrm{D})}$ (4)

$\Delta E_{\mathrm{TD}(\mathrm{A}) \text {-hybrid }}$ can either be based on a global or range-separated DHDF description. 1721 An alternative SCS/SOS-DHDF definition in which the SCS/SOS-CIS(D) part is split into short- and long-range components has been recently suggested ${ }^{35}$ but cannot be tested herein for technical reasons. Instead, we focus on TD(A)-DHDFs as defined above.

\section{Computational details}

A local version of ORCA 4.2.1 $1^{41,43}$ was used to calculate the doublet-doublet excitation energies for the radical set. A total of 29 functionals were assessed, as listed in Table1. All functionals assessed herein are available in the newly released ORCA 5. As explained in Ref. 21, the SCS fit for B2PLYP and $\omega$ B2PLYP led to the SOS variant. Herein, we use the shorthand notation "SOS-B2PLYP" and "SOS- $\omega$ B2PLYP" to refer to these two functionals - which are labeled SCS/SOS-B2PLYP21 and SCS/SOS$\omega B 2 P L Y P 21$, respectively, in Ref. 21 .

For all calculations the SCF convergence criterion was set to $10^{-8} \mathrm{E}_{\mathrm{h}}$, and ORCA's numerical quadrature grid "5" and "finalgrid 6" were used. The resolution of the identity approximation was used with appropriate auxiliary basis sets for the perturbative parts of DHDFs.

Pre-optimized UCCSD $(T)^{44} /$ aug-cc-pVTZ ${ }^{45 / 47}$ ground-state geometries from Ref. 38 were used. UCC 3 38 4849/aug-cc-PVTZ excitation energies from Ref. 38 were used as reference energies in the statistical evaluation. The aug-cc-pVTZ basis set was used for all calculations presented herein to enable a direct comparison to the reference values. Two transitions listed in Ref. 38 were not analyzed in this paper due to some excited states being inexplicably missing when calculated with ORCA. These are the ${ }^{2} \Sigma^{+}$transition of the $\mathrm{CH}$ radical and the second ${ }^{2} \Pi$ transition of the $\mathrm{BeH}$ radical. We would also like to point out that the tested systems are challenging and the automated assessment of all 29 functionals was not always straightfor-
Table 1: List of assessed functionals. Global and range-separated methods appearing in the same row are related.

\begin{tabular}{|c|c|c|}
\hline & global & range-separated \\
\hline hybrid & $\begin{array}{l}\text { B3LYP } 5051] \\
\text { BHLYP } \\
\text { PBE0 } 53554\end{array}$ & CAM-B3LYP \\
\hline double-hybrid & $\begin{array}{l}\text { B2PLYP }{ }^{9} \\
\text { SOS-B2PLYP } 21] \\
\text { B2GP-PLYP } 56 \\
\text { SCS-B2GP-PLYP } \\
\text { SOS-B2GP-PLYP } \\
\text { PBE0-DH }{ }^{[7]} \\
\text { PBE-QIDH } 51 \\
\text { SCS-PBE-QIDH } \\
\text { SOS-PBE-QIDH }\end{array}$ & 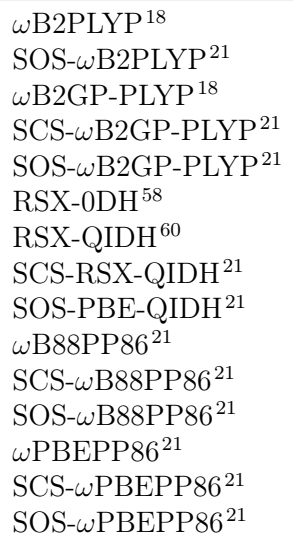 \\
\hline
\end{tabular}

ward, for instance due to swapping of states or the emergence of very close-lying states for some DFAs. In some instances, UCCSD energies from Ref. 38 had to be reproduced to obtain information about the correct orbital contributions. A very challenging excitation that showed multiple problems of this nature is the ${ }^{2} \Sigma^{+}$transition in $\mathrm{CO}^{+}$. As we will discuss later, it also showed large double-excitation character and due to our observed problems we also excluded this state from our analysis. The complete set analyzed herein, thus, comprises 45 excitations.

$\% \mathrm{~T} 1$ values were obtained with UCCSD calculations where necessary.

\section{Results and Discussion}

We separate the discussion of our results into five brief sections. First, we analyze the differences between the use of TD- and TDADFT for the functionals tested. Then, we look at the effects of range-separation and the impact of spin-component/spin-opposite scaling on DHDFs. Following that, we compare DHDFs against conventional hybrids and comment on the robustness of particular functionals. Those first four sections comprise our re- 


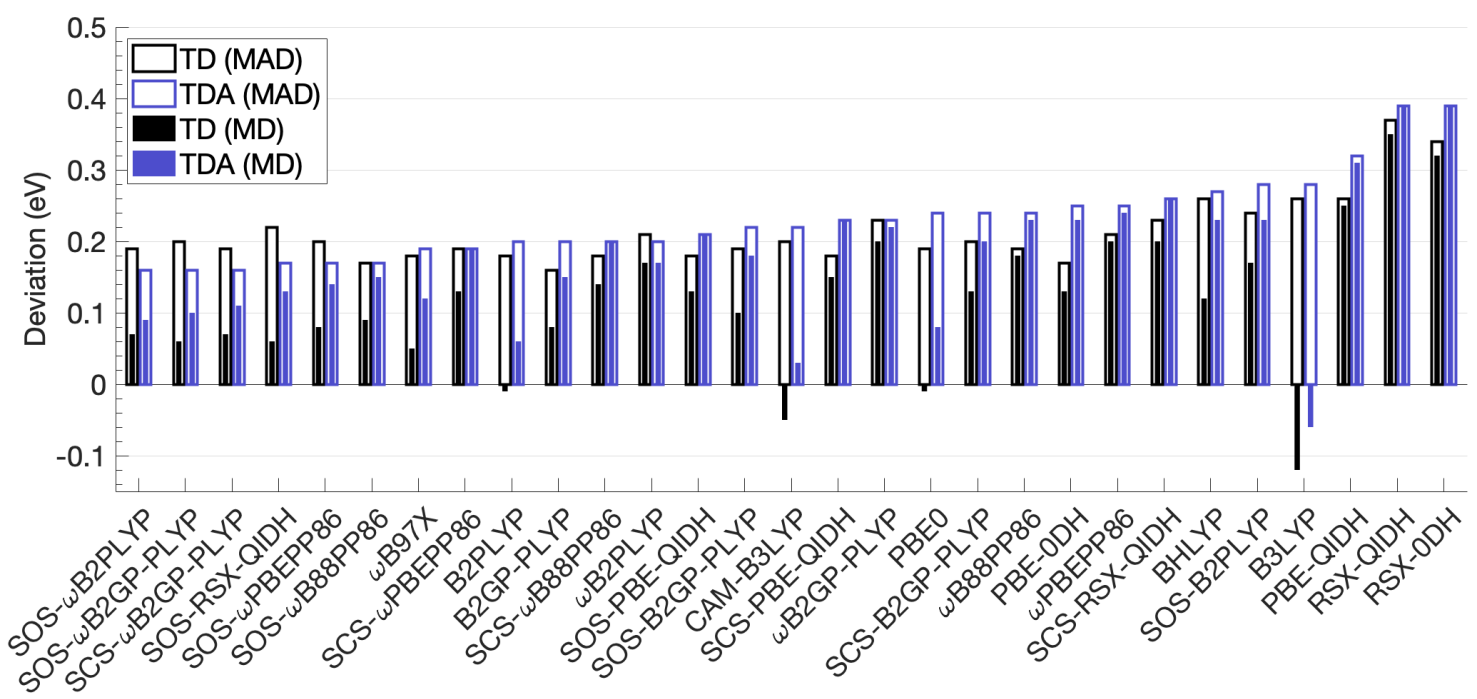

Figure 2: MDs and MADs (in eV) averaged over 45 doublet-doublet excitation energies using TDDFT and TDA-DFT for all tested functionals, ranked by TDA MAD, followed by RMSDs and then absolute MDs in case of identical MADs.

sults for all 45 excitations. The discussion then concludes with a second analysis that excludes 4 states due to high double excitation or multireference character. Throughout our discussion, a deviation is defined as the difference between the DFA and reference value, which means that negative deviations and mean deviations (MDs) stand for underestimated excitation energies.Individual excitations for each DFA as well as all statistical metrics - including root mean square deviations (RMSDs), minimum and maximum deviations, and deviation spans - are available in the Supporting Information (SI).

\subsection{On the Tamm-Dancoff ap- proximation}

The TDA is attractive to computational chemists not only for its improved computational efficiency over TD-DFT, but also because it is known to overcome triplet instability issues. $\frac{61}{64}$ As a result, TDA-DFT frequently produces more accurate singlet-triplet excitation energies than TD-DFT, which is also true for DHDFs. ${ }^{19121}$ Herein, we intend to determine whether this trend also applies to doublet-doublet excitations.

In Figure 2 we present the MDs and mean absolute deviations (MADs) of the 29 DFAs tested with TD- and TDA-DFT for the entire set of 45 excitations. Immediately notable is that doublet-doublet excitations are not subject to instability issues. $\omega$ B2PLYP's MD is the only one not affected by the TDA. For all other functionals, there is an increase in $\mathrm{MD}$ from TD-DFT to TDA, ranging from $0.02 \mathrm{eV}$ to 0.11 $\mathrm{eV}$. The smallest MD increase is from TD-SOS$\omega$ B2PLYP to TDA-SOS- $\omega$ B2PLYP (from 0.07 $\mathrm{eV}$ to $0.09 \mathrm{eV}$ ) and from TD- $\omega \mathrm{B} 2 \mathrm{GP}-\mathrm{PLYP}$ to TDA- $\omega$ B2GP-PLYP (from 0.20 to $0.22 \mathrm{eV}$ ). At the other end, the MD of BHLYP increases from $0.12 \mathrm{eV}$ to $0.23 \mathrm{eV}$ for the TDA. The increased MDs indicate that the TDA causes a systematic blueshift in excitation energies across the entire radical set, which is a known trend for other excitation types. ${ }^{19 \mid 30}$ While this is unfavorable for most functionals, this blueshift improves some MDs; for instance, the blueshift results in an MD of $-0.06 \mathrm{eV}$ for TDA-B3LYP, which is more favourable than the $-0.12 \mathrm{eV}$ obtained with TD-B3LYP. Despite this, the MAD of TDA-B3LYP is by $0.02 \mathrm{eV}$ higher than TDB3LYP. This indicates that the set contains examples of already blueshifted excitation energies at the TD-DFT level, whose deviations are exacerbated due to the additional blueshift from the TDA.

Interestingly, an increase in $\mathrm{MD}$ can still coincide with reduced deviation spans. For in- 
stance, despite an increase of $0.11 \mathrm{eV}$ in the MD when going from TD- to TDA-BHLYP, we observe a decrease in the deviation span of 1.01 $\mathrm{eV}$ (see SI). Other notable reductions in the deviation span of about $0.5 \mathrm{eV}$ or more for the TDA are observed for B2PLYP, SOS-B2PLYP, $\omega \mathrm{B} 2 \mathrm{PLYP}$, and SOS- $\omega$ B2PLYP. While deviation spans are only marginally affected by the TDA for a large number of functionals, the largest increase of $0.16 \mathrm{eV}$ is observed for B2GPPLYP (see SI).

In most cases, the systematic blueshift observed with a TDA approach also corresponds to an increase in MAD, however, with all increases being slightly under the chemicalaccuracy threshold of $0.1 \mathrm{eV} .6[65$ There are some notable exceptions: all variations of $\omega$ B2PLYP have slightly lower MADs when using the TDA (decreases in MAD of $0.03 \mathrm{eV}$ and $0.01 \mathrm{eV}$ for SOS- $\omega$ B2PLYP and $\omega$ B2PLYP, respectively). Employing the TDA also decreases MADs for SCS/SOS- $\omega$ B2GP-PLYP, SOS-RSX-QIDH, and SOS- $\omega$ PBEPP86. No MAD changes are observed for $\omega$ B2GP-PLYP, SOS- $\omega$ B88PP86, and SCS- $\omega$ PBEPP86. Across the TDA results, the lowest MAD is $0.16 \mathrm{eV}$ for SCS- and SOS$\omega$ B2GP-PLYP as well as SOS- $\omega$ B2PLYP. This is also the lowest-found MAD value across the entire set of 45 excitations and all assessed functionals. SOS-RSX-QIDH, SOS- $\omega$ B88PP86, and SOS- $\omega$ PBEPP86 perform similarly, with MADs of $0.17 \mathrm{eV}$. At $0.19 \mathrm{eV}$, SCS- $\omega$ PBEPP86 is the only remaining TDA-DHDF with an MAD below $0.2 \mathrm{eV}$.

Considering previous works that show that TD-DFT is unsuitable for singlet-triplet excitations, ${ }^{61 / 63[64] 66}$ the performance of these TDADHDFs makes them attractive for the calculation of doublet-doublet excitations. For the remainder of the analysis in this paper we refer to only TDA excitation energies unless explicitly specified otherwise. The full set of TD- and TDA-DFT data is available in the SI.

\subsection{Effects of range-separation}

The next aspect we investigate is rangeseparation. The MDs and MADs of five global, unscaled DFAs and their range-separated coun-

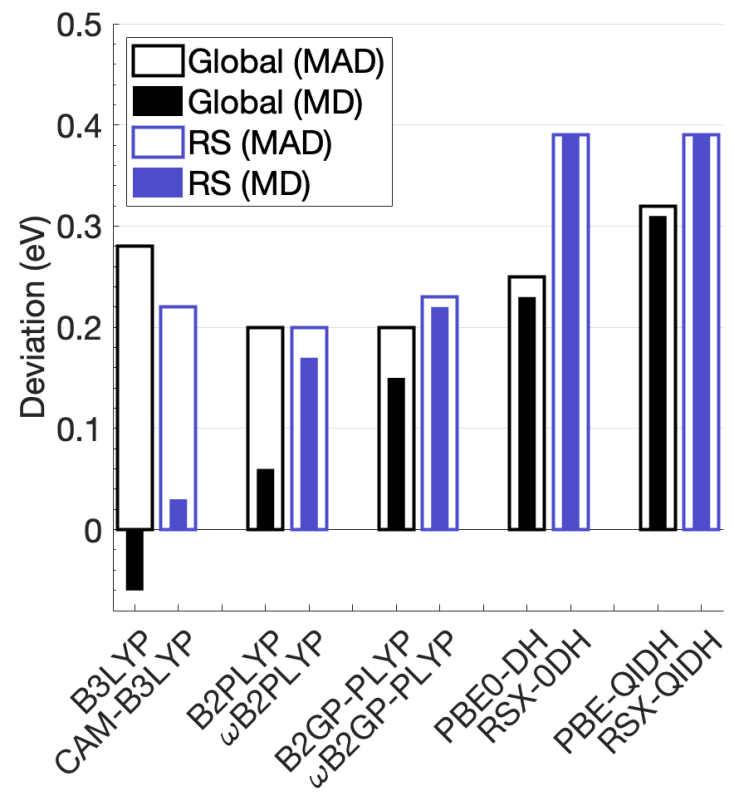

Figure 3: MDs and MADs (in eV) for global and range-separated (RS) functionals using TDADFT.

terparts are presented in Fig. 3. In all of these cases, the range-separated functionals have more positive MDs than the global counterparts. Systematic blueshift from rangeseparation is a known effect for DFAs, including DHDFs. $\frac{8|18| 19 \mid 30}{\omega B}$ 2GP-PLYP is the least blueshifted by range-separation (an increase in $\mathrm{MD}$ of $0.07 \mathrm{eV}$ from B2GP-PLYP to $\omega$ B2GPPLYP), and RSX-0DH shows the largest MD increase of $0.16 \mathrm{eV}$ (from PBE0-DH to RSX0DH).

For three of the four herein tested, unscaled range-separated DHDFs ( $\omega$ B2GP-PLYP, RSX0DH, and RSX-QIDH), the MADs are larger than for their global counterparts. Of these, the smallest increase is $0.03 \mathrm{eV}$ for $\omega \mathrm{B} 2 \mathrm{GP}-\mathrm{PLYP}$ and the largest increase, $0.14 \mathrm{eV}$, is again for RSX-0DH. B2PLYP and $\omega$ B2PLYP share the same MAD. Interestingly, the range-separated hybrid CAM-B3LYP does not follow this trend. The MAD for CAM-B3LYP is $0.22 \mathrm{eV}$ and therefore lower than the MAD of $0.28 \mathrm{eV}$ for B3LYP. In this case, range-separation improves the performance of the underlying functional, which may be due to the inclusion of $65 \%$ Fock exchange at long-range compared to $100 \%$ for the other range-separated functionals.

The trends observed for range-separation be- 

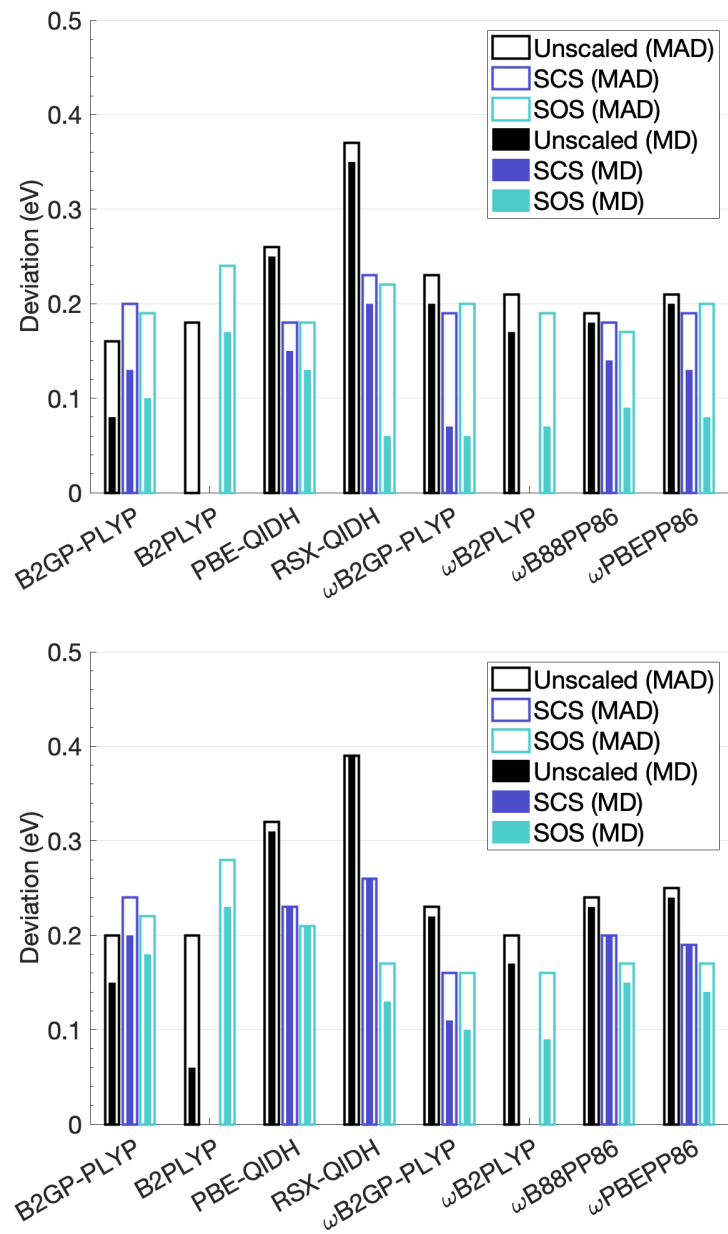

Figure 4: MDs and MADs (in eV) for scaled and unscaled functionals. Top: TD-DFT. Bottom: TDA-DFT.

come more complex when we introduce spincomponent and spin-opposite scaling, where we observe reductions of up to $0.14 \mathrm{eV}$ in $\mathrm{MD}$ and $0.12 \mathrm{eV}$ in MAD (from SOS-B2PLYP to SOS- $\omega$ B2PLYP) and the only marginal increase is seen when moving from SCS-PBE-QIDH to SCS-RSX-QIDH $(0.03 \mathrm{eV}$ both for MD and MAD) (see SI). The impact of SCS and SOS is discussed in more detail in the following section.

\subsection{Impact of spin-component and spin-opposite scaling}

The effects of SCS and SOS on the MDs and MADs of 8 unscaled parent DFAs are illustrated in Fig. 4. For the vast majority of DFAs, SCS/SOS results in MDs and MADs closer to zero compared to the unscaled version of each functional, and these trends are nearly the same for both the TD-DFT (Fig. 4, top) and TDADFT schemes (Fig. 4, bottom). The global B2GP-PLYP and B2PLYP functionals are exceptions to this trend; these DFAs perform better when unscaled. This is especially notable for the latter functional-TDA-SOS-B2PLYP has a higher $\mathrm{MD}$ and MAD (increases of $0.17 \mathrm{eV}$ and $0.08 \mathrm{eV}$, respectively) than unscaled TDAB2PLYP.

Improvements in MADs from SCS compared to an unscaled DFA range from being marginal (0.01 eV for TD-SCS- $\omega$ B88PP86) to significant (0.14 eV for TD-SCS-RSX-QIDH). Improvements in the same range are also observed for most SOS methods albeit with a much larger maximum improvement (0.22 eV for TDA-SOSRSX-QIDH). In the overwhelming number of cases, the SCS variant of a DFA results in higher MDs (and thus more blueshifted excitation energies) than the SOS version. Usually this also coincides with the SCS variants having slightly higher MADs than the SOS variant, with TDA- $\omega$ B2GP-PLYP being a notable exception due to having the same MADs. The observed trends are the same for full TD-DFT and TDA-DFT, with the full TD-DFT versions of SOS- $\omega$ PBEPP86 and SOS- $\omega$ B2GP-PLYP being exceptions because they have slightly higher MADs than their SCS counterparts, contrary to their TDA-DFT versions (Fig. 4). The impact of SCS and SOS compared to the unscaled version is the highest for TD/TDA-RSX-QIDH. Also the difference between SCS and SOS is the highest for this functional compared to the other tested DFAs.

Returning to range-separation we see that when SCS/SOS is introduced, some of the range-separated DFAs perform better than the global functionals they are based on. While scaling yields worse results for B2(GP-)PLYP, the scaled variants of range-separated $\omega \mathrm{B} 2 \mathrm{GP}-$ PLYP and $\omega$ B2PLYP have lower MDs and MADs than the unscaled range-separated counterparts (Fig. 4). This again indicates a beneficial interplay between SCS/SOS and rangeseparation for some of the new $\mathrm{TD}(\mathrm{A})-\mathrm{DHDFs}$, as also reported for closed-shell systems in Ref. 21 . 


\subsection{Comparison of all functionals tested for the entire set}

We continue our discussion of the entire set of 45 excitations with a comprehensive comparison between all tested approaches, which combines the three different aspects that we have individually addressed in the preceding sections. All results are shown in Fig. 2. When ranking the assessed DFAs we consider the MADs first, followed by RMSDs and absolute MDs when necessary. It is known that DHDFs are the most accurate DFAs for ground-state calculations, ${ }_{10167-70}$ as well as singlet-singlet and singlet-triplet excitation en-

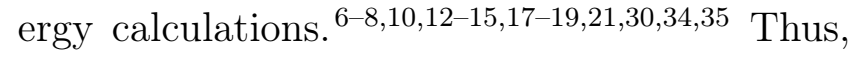
it would be reasonable to expect that this would also be true for doublet-doublet excitations. While the best-performing DFAs for the radical test set are indeed DHDFs, as discussed below, the assessed hybrid functionals show comparable performance to some of the DHDFs tested. For instance, TD- $\omega$ B97X $(\mathrm{MAD}=0.18 \mathrm{eV})$ is the eleventh-best of all tested approaches when TD- and TDA-DFT results are combined and ranked after the above-mentioned criteria; it is the fourth-best among all full TD-DFT methods. Unexpectedly, the worst-performing functionals are also DHDFs, which highlights the challenging nature of doublet-doublet excitation energy calculations. A total of six DHDF-based approaches have the same or worse MAD than the worst hybrid approach TDA-B3LYP $(\mathrm{MAD}=0.28$ $\mathrm{eV}$ ), namely TDA-SOS-B2PLYP, TDA-PBEQIDH, TD/TDA-RSX-0DH, and TD/TDARSX-QIDH.

For all assessed approaches, MADs range from $0.16 \mathrm{eV}$ (TD-B2GP-PLYP, TDA-SOS$\omega$ B2PLYP, and TDA-SCS/SOS- $\omega$ B2GPPLYP) to $0.39 \mathrm{eV}$ (TDA-RSX-QIDH and TDARSX-0DH), once again confirming that socalled 'non-empirical' DHDFs are not automatically superior to semi-empirical ones; for other examples of this finding, see e.g. Refs 10,19,21,71 and 72. With MADs of 0.17 or $0.18 \mathrm{eV}$, the following DHDFs are very close to the best DFAs: TD-PBE0-DH, TDA-SOSRSX-QIDH, TDA-SOS- $\omega$ PBEPP86, TD/TDA-
SOS- $\omega$ B88PP86, TD-B2PLYP, TD-SOS/SCSPBE-QIDH, and TD-SCS- $\omega$ PBEPP86.

When considering that TDA-DHDFs are more efficient for larger systems, we notice that TDA-DHDFs with SCS/SOS and rangeseparation are among the best-performing methods, which parallels findings for various local and long-range singlet-singlet and singlettriplet excitations. ${ }^{21}$ That being said, we notice some discrepancies with our detailed recommendations from previous studies and also some unusually large blueshifts of $1 \mathrm{eV}$ or more even for many of the usually best-performing TD(A)-DHDFs. The potential reason for this will be discussed in the next section, which will provide an updated analysis.

\subsection{Analysis after excluding double-excitation states}

One well-known flaw of TD(A)-DFT is its inability to describe transitions with high doubleexcitation character due to the adiabatic approximation. ${ }^{7374} \mathrm{TD}(\mathrm{A})$-DHDFs are no exception to this and in fact their high amount of Fock exchange can further increase deviations. ${ }^{61721}$ Upon closer inspection of the WFT data in the original paper ${ }^{38}$ that published the herein assessed radical set and after additionally conducted UCCSD calculations of systems with deviations of more than $0.5 \mathrm{eV}$ for DHDFs that are known to usually perform well, we identified excitations with high doubleexcitation or multi-reference character. The relevant systems and states that caught our attention after such an analysis are $\mathrm{CNO}\left(\right.$ state $\left.^{2} \Sigma^{+}\right)$, $\mathrm{CO}^{+}\left(\right.$states $\left.{ }^{2} \Pi\right)$ and $\mathrm{NCO}\left(\right.$ states $^{2} \Sigma^{+}$and $\left.{ }^{2} \Pi\right)$.

Our reasoning for $\mathrm{CNO}$ and $\mathrm{CO}^{+}$is based on the \% 11 diagnostic for UCCSD and large deviations of the latter from UCC3 numbers published in Ref. 38, which coincide also with large outliers for assessed TD(A)-DHDFs in this work. A \% 1 1 diagnostic under 90 was used to identify problematic excitations. The reported ${ }^{2} \Sigma^{+}$excitation energy for CNO at the UCCSD level is $2.24 \mathrm{eV}(\% \mathrm{~T} 1=88.00)$, which represents a deviation of $0.49 \mathrm{eV}$ compared to UCC3. Similarly, many (but not all) DHDFs have large deviations between $0.99 \mathrm{eV}$ (TD-PBE-QIDH) 


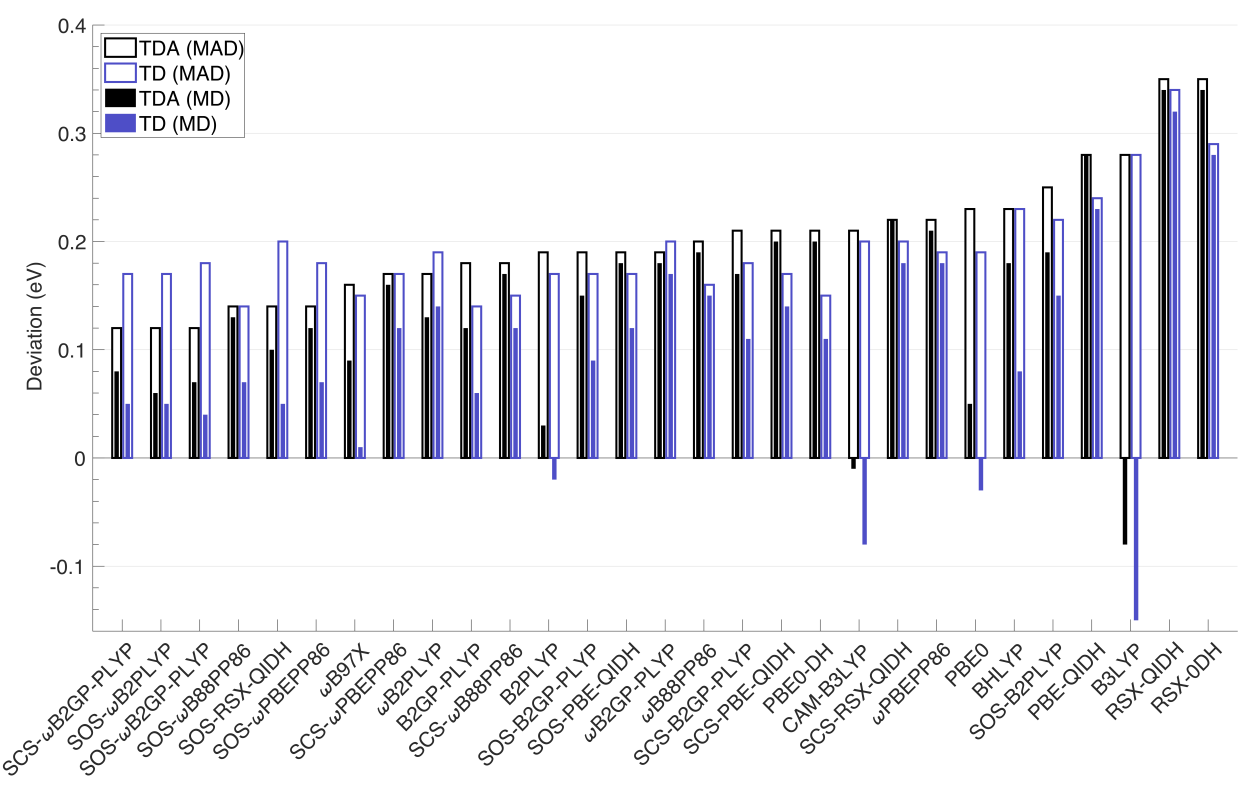

Figure 5: MDs and MADs (in eV) for the reduced set of 41 doublet-doublet excitation energies using TD-DFT and TDA-DFT for all tested functionals, ranked by TDA MAD, followed by RMSDs and then absolute MDs in case of identical MADs.

and $1.35 \mathrm{eV}$ (TDA-RSX-QIDH) The UCCSD excitation energy for the ${ }^{2} \Pi$ transition in $\mathrm{CO}^{+}$ is $3.60(\% \mathrm{~T} 1=87.79)$, which is why it was ruled out, too. Note that we had already discarded the ${ }^{2} \Sigma^{+}$transition of the same molecule due to problems with this state. Its \% 1 is 87.96 and its double excitation character might be a potential answer for the problems we alluded to in Section 3 .

In the case of $\mathrm{NCO}$, we note that the ${ }^{2} \Sigma^{+}$ and ${ }^{2} \Pi$ transitions are mainly dominated by singly excited configurations $(\% \mathrm{~T} 1=93.85$ and $\% \mathrm{~T} 1=90.96)$. Despite that, Loos et al. determined that EOM-CCSDTQ ${ }^{75}$ calculations were mandatory to obtain reliable transitions, particularly for the ${ }^{2} \Pi$ state, and that they changed even more for MRCI + Q. ${ }^{38}$ Because we cannot fully guarantee the accuracy of the reference or potential problems of the methods for such multi-reference cases, NCO was also regarded as a problematic case in this study.

Due to known inadequacies of $\mathrm{TD}(\mathrm{A})$ DFT for closed-shell systems with similar difficulties - double-excitation or high multireference character-previous TD(A)-DHDF studies have presented two separate sets of statistics for the benchmark sets they were in- vestigating, namely with the challenging transitions included and excluded. ${ }^{21 \mid 76}$ Here, we follow the same principle. While the previous section provided us with the statistics over 45 excitations of the entire set, this section presents a brief discussion of a reduced set with 41 excitations for 21 radicals. The statistical data for this slightly reduced set are provided in the SI and the relevant results are shown in Fig. 5. While a recent double-hybrid approach based on the second-order algebraicdiagrammatic construction ${ }^{77}[\mathrm{ADC}(2)]$ looked promising for double excitations in closed-shell systems, $\frac{176}{1}$ that advantage is expected to also come at a higher computational cost, but could be pursued in a future study.

A direct comparison between Fig. 5 and Fig. 2 shows that there is a systematic improvement for the tested methods regardless of choosing the TD- or TDA-DFT scheme. The average MAD over all tested TD- and TDA-DFT approaches is $0.22 \mathrm{eV}$ for the complete set and reduced to $0.20 \mathrm{eV}$ after excluding the four difficult states. MADs now range from $0.12 \mathrm{eV}$ to $0.35 \mathrm{eV}$ (a $0.04 \mathrm{eV}$ improvement at both ends of the spectrum). Most importantly, the best methods are now closer to the chemical accu- 
racy threshold of $0.1 \mathrm{eV}$.

While there is overall little substantial change to the DFA ranking, we do notice a shift when comparing the best TDA- with the best TDDFT methods. The six best approaches are now exclusively TDA-DHDFs with SCS/SOS and range-separation, namely SCS- $\omega$ B2GP-PLYP, SOS- $\omega$ B2PLYP, and SOS- $\omega$ B2GP-PLYP with MADs of $0.12 \mathrm{eV}$, followed by SOS- $\omega$ B88PP86, SOS-RSX-QIDH, and SOS- $\omega$ PBEPP86 with MADs of $0.14 \mathrm{eV}$. The first full TD-DFT method in our ranking is TD-B2GP-PLYP with an MAD of $0.14 \mathrm{eV}$ but a slightly higher RMSD than the preceding three TDA-DHDFs. B3LYP remains to be the worst hybrid functional and four DHDFs are still worse, namely the TD and TDA versions of RSX-0DH and RSX-QIDH.

UCCSD has an MAD of $0.12 \mathrm{eV}$ based on the results taken from Ref. 38, which is the same as for the best three DFAs, however, it seems to have a more systematic blueshift due to an MD with exactly the same value. The top-three DFAs all have MDs closer to zero and therefore outperform CCSD on average. Therefore, we warn against using CCSD or approximate versions of it as a reference for benchmarking TD(A)-DHDFs.

Using the TDA and SOS can both provide computational advantages, which makes the top-six DFAs in this study quite attractive for larger systems. Our findings for the best-performing methods mirror our previous benchmark studies on singlet-singlet and singlet-triplet excitations, with the difference that SOS- $\omega$ B88PP86 and SOS- $\omega$ PBEPP86 performed better for those than the top-three functional in the present study. Given that their MADs for doublet-doublet excitations are only marginally larger than for the top-three methods, we can recommend SOS- $\omega$ B88PP86 and SOS- $\omega$ PBEPP86 as more robust methods that work well for different types of excitations.

However, despite the success of DHDFs with range-separation and SCS/SOS for doubletdoublet excitations, it is important to note that even the best functionals are still slightly above the chemical accuracy threshold and more development in this area might be warranted.

\section{Summary and conclusions}

In this work, we used a range of hybrid and double-hybrid (DHDF) density functionals to calculate doublet-doublet excitation energies in small radicals. The study of open-shell systems with time-dependent DHDFs was the first detailed of this kind, and our aim was to shed light on the current state-of-the-art techniques in the field and to clarify if there is still room for future improvements. The performance of 29 functionals was evaluated by comparing the calculated excitation energies against UCC3/augcc-PVTZ reference energies from Ref. 38.

First we investigated the Tamm-Dancoff approximation (TDA) and its effects on doubletdoublet transitions. Our findings indicate that doublet-doublet excitations are not subject to TD-DFT instability problems. The TDA was found to cause a systematic blueshift in excitation energies, and-unlike for triplet excitations - it slightly worsened the performance of most functionals except for the bestperforming ones.

To investigate the effects of range-separation, we compared range-separated functionals against global ones. Range-separated double hybrids resulted in larger mean absolute deviations (MADs) than their global counterparts unless spin-component/spin-opposite scaling (SCS/SOS) was applied.

Introducing the effects of SCS/SOS revealed that many of the scaled DHDFs performed better with range-separation. All rangeseparated DHDFs delivered lower MADs with SCS/SOS, however the results for the global B2(GP-)PLYP functionals were worsened with SCS/SOS. DHDFs did not categorically outperform hybrid functionals: while the bestperforming DFAs were indeed DHDFs, the worst-performing functionals also belonged to the fifth rung of Jacob's Ladder, more specifically to the class of so-called 'non-empirical' DHDFs. Thus, once again it has been demonstrated how those were outperformed by semiempirical DHDFs despite persisting claims of the opposite.

Our analysis revealed four difficult states due to high double-excitation or multi-reference 
character and after excluding those systems, we noticed improvements for all tested functionals. The six best methods in our study had MADs of $0.12 \mathrm{eV}$ and $0.14 \mathrm{eV}$, respectively, and they all have in common that they are spin-component or spin-opposite scaled double hybrids combined with range separation and applied within the TDA. More specifically, these methods are: SCS- $\omega$ B2GP-PLYP, SOS- $\omega$ B2PLYP, SOS$\omega B 2 G P-P L Y P, \quad$ SOS- $\omega B 88 P P 86, \quad$ SOS-RSXQIDH, and SOS- $\omega$ PBEPP86. It is noteworthy that those methods worked without further optimization of their parameters, which had been determined on closed-shell systems. Given their better performance for singlet-singlet and singlet-triplet excitations, we recommend TDASOS- $\omega$ B88PP86 and TDA-SOS- $\omega$ PBEPP86 as robust methods for general excitation energies until further improvements have been achieved that surpass the chemical accuracy target, particularly for challenging systems, without increasing the computational effort.

Acknowledgement M. C.-P. acknowledges a Melbourne Research Scholarship from late 2017 to mid-2021. L. G. is grateful for generous allocations of computational resources from the National Computational Infrastructure (NCI) Facility within the National Computational Merit Allocation Scheme (project fk5), and Research Platform Services (ResPlat) at The University of Melbourne (project punim0094).

\section{Notes}

The authors declare no competing financial interest.

\section{Supporting Information Avail- able}

The SI contains all excitation energies for all assessed functionals as well as their detailed statistics.

\section{References}

(1) Hohenberg, P.; Kohn, W. Inhomogeneous electron gas. Phys. Rev. B 1964, 136, 864-871.

(2) Kohn, W.; Sham, L. J. Self-consistent equations including exchange and correlation effects. Phys. Rev. 1965, 140, A1133A1138.

(3) Runge, E.; Gross, E. K. U. Densityfunctional theory for time-dependent systems. Phys. Rev. Lett. 1984, 52, 9971000 .

(4) Hirata, S.; Head-Gordon, M. Timedependent density functional theory within the Tamm-Dancoff approximation. Chem. Phys. Lett. 1999, 314, 291-299.

(5) Perdew, J. P.; Schmidt, K. Jacob's ladder of density functional approximations for the exchange-correlation energy. AIP Conference Proceedings 2001, 577, 1-20.

(6) Goerigk, L.; Moellmann, J.; Grimme, S. Computation of accurate excitation energies for large organic molecules with double-hybrid density functionals. Phys. Chem. Chem. Phys. 2009, 11, 4611-4620.

(7) Goerigk, L.; Grimme, S. Assessment of TD-DFT methods and of various spin scaled CIS(D) and CC2 versions for the treatment of low-lying valence excitations of large organic dyes. J. Chem. Phys. 2010, 132, 184103.

(8) Goerigk, L.; Casanova-Paéz, M. The trip to the density functional theory zoo continues: making a case for time-dependent double hybrids for excited-state problems. Aust. J. Chem. 2021, 74, 3-15.

(9) Grimme, S. Semiempirical hybrid functional with perturbative second-order correlation. J. Chem. Phys. 2006, 124, 034108 .

(10) Goerigk, L.; Grimme, S. Double-hybrid density functionals. Wiley Interdiscip. Rev. Comput. Mol. Sci. 2014, 4, 576-600. 
(11) Grimme, S.; Neese, F. Double-hybrid density functional theory for excited electronic states of molecules. J. Chem. Phys. 2007, 12\%, 154116.

(12) Goerigk, L.; Grimme, S. Calculation of electronic circular dichroism spectra with time-dependent double-hybrid density functional theory. J. Phys. Chem. A 2009, 113, 767-776.

(13) Goerigk, L.; Grimme, S. Double-hybrid density functionals provide a balanced description of excited ${ }^{1} \mathrm{~L}_{a}$ and ${ }^{1} \mathrm{~L}_{b}$ states in polycyclic aromatic hydrocarbons. $J$. Chem. Theory Comput. 2011, 7, 32723277.

(14) Goerigk, L.; Kruse, H.; Grimme, S. Comprehensive chiroptical spectroscopy; Wiley-Blackwell, 2012; pp 643-673.

(15) Meo, F. D.; Trouillas, P.; Adamo, C.; Sancho-García, J. C. Application of recent double-hybrid density functionals to lowlying singlet-singlet excitation energies of large organic compounds. J. Chem. Phys. 2013, 139, 164104.

(16) Momeni, M. R.; Brown, A. A local CC2 and TDA-DFT double hybrid study on BODIPY/aza-BODIPY dimers as heavy atom free triplet photosensitizers for photodynamic therapy applications. J. Phys. Chem. A 2016, 120, 2550-2560.

(17) Schwabe, T.; Goerigk, L. Time-dependent double-hybrid density functionals with spin-component and spin-opposite scaling. J. Chem. Theory Comput. 2017, 13, 4307-4323.

(18) Casanova-Páez, M.; Dardis, M. B.; Goerigk, L. $\omega$ B2PLYP and $\omega$ B2GPPLYP: The first two double-hybrid density functionals with long-range correction optimized for excitation energies. J. Chem. Theory. Comput. 2019, 15, 4735-4744.

(19) Casanova-Páez, M.; Goerigk, L. Assessing the Tamm-Dancoff approximation, singlet-singlet, and singlet-triplet excitations with the latest long-range corrected double-hybrid density functionals. J. Chem. Phys. 2020, 153, 064106.

(20) Goerigk, L.; Grimme, S. Quantum chemical investigation of exciton coupling: super-molecular calculations of a merocyanine dimer aggregate. ChemPhysChem 2008, 9, 2467-2470.

(21) Casanova-Páez, M.; Goerigk, L. Timedependent long-range-corrected doublehybrid density functionals with spincomponent and spin-opposite scaling: a comprehensive analysis of singlet-singlet and singlet-triplet excitation energies. $J$. Chem. Theory Comput. 2021, 17, 51655186 .

(22) Grimme, S. Improved second-order møller-plesset perturbation theory by separate scaling of parallel- and antiparallel-spin pair correlation energies. J. Chem. Phys. 2003, 118, 9095-9102.

(23) Jung, Y.; Lochan, R. C.; Dutoi, A. D.; Head-Gordon, M. Scaled opposite-spin second order Møller-Plesset correlation energy: an economical electronic structure method. J. Chem. Phys. 2004, 121, 97939802 .

(24) Rhee, Y. M.; Head-Gordon, M. Scaled second-order perturbation corrections to configuration interaction singles: efficient and reliable excitation energy methods. $J$. Phys. Chem. A 2007, 111, 5314-5326.

(25) Grimme, S.; Goerigk, L.; Fink, R. F. Spin-component-scaled electron correlation methods. Wiley Interdiscip. Rev.: Comput. Mol. Sci. 2012, 2, 886-906.

(26) Leininger, T.; Stoll, H.; Werner, H.-J.; Savin, A. Combining long-range configuration interaction with short-range density functionals. Chem. Phys. Lett. 1997, 275, $151-160$.

(27) Iikura, H.; Tsuneda, T.; Yanai, T.; Hirao, K. A long-range correction scheme 
for generalized-gradient-approximation exchange functionals. J. Chem. Phys. 2001, 115, 3540-3544.

(28) Yanai, T.; Tew, D. P.; Handy, N. C. A new hybrid exchange-correlation functional using the Coulomb-attenuating method (CAM-B3LYP). Chem. Phys. Lett. 2004, 393, 51-57.

(29) Baer, R.; Neuhauser, D. Density functional theory with correct long-range asymptotic behavior. Phys. Rev. Lett. 2005, 94, 043002.

(30) Casanova-Páez, M.; Goerigk, L. Global double hybrids do not work for charge transfer: A comment on "Double hybrids and time-dependent density functional theory: An implementation and benchmark on charge transfer excited states". $J$. Comput. Chem. 2021, 42, 528-533.

(31) Tozer, D. J.; Amos, R. D.; Handy, N. C.; Roos, B. O.; Serrano-Andrés, L. Does density functional theory contribute to the understanding of excited states of unsaturated organic compounds? Mol. Phys. 1999, 97, 859-868.

(32) Tozer, D. J. Relationship between long-range charge-transfer excitation energy error and integer discontinuity in Kohn-Sham theory. J. Chem. Phys. 2003, 119, 12697-12699.

(33) Dreuw, A.; Head-Gordon, M. Failure of time-dependent density functional theory for long-range charge-transfer excited states:the zincbacteriochlorinbacteriochlorin and bacteriochlorophyllspheroidene complexes. J. Am. Chem. Soc. 2004, 126, 4007-4016.

(34) Mester, D.; Kállay, M. A simple rangeseparated double-hybrid density functional theory for excited states. J. Chem. Theory Comput. 2021, 17, 927-942.

(35) Mester, D.; Kállay, M. Spin-scaled rangeseparated double-hybrid Density Func- tional Theory for excited states. J. Chem. Theory Comput. 2021, 17, 4211-4224.

(36) Hernández-Martínez, L.; Brémond, E.; Pérez-Jiménez, A. J.; San-Fabián, E.; Adamo, C.; Sancho-García, J. C. Nonempirical (double-hybrid) density functionals applied to atomic excitation energies: a systematic basis set investigation. Int. J. Quantum Chem. 2020, 120, e26193.

(37) Brémond, E.; Ottochian, A.; PérezJiménez, A. J.; Ciofini, I.; Scalmani, G.; Frisch, M. J.; Sancho-García, J. C.; Adamo, C. Assessing challenging intraand inter-molecular charge-transfer excitations energies with double-hybrid density functionals. J. Comput. Chem. 2021, 42, 970-981.

(38) Loos, P.-F.; Scemama, A.; BoggioPasqua, M.; Jacquemin, D. Mountaineering strategy to excited states: highly accurate energies and benchmarks for exotic molecules and radicals. J. Chem. Theory Comput. 2020, 16, 3720-3736.

(39) McLachlan, A. D.; Ball, M. A. Timedependent Hartree-Fock theory for molecules. Rev. Mod. Phys. 1964, 36, 844-855.

(40) Head-Gordon, M.; Rico, R. J.; Oumi, M.; Lee, T. J. A doubles correction to electronic excited states from configuration interaction in the space of single substitutions. Chemical Physics Letters 1994, 219, 21-29.

(41) Neese, F. The ORCA program system. Wiley Interdiscip. Rev.: Comput. Mol. Sci. 2012, 2, 73-78.

(42) Neese, F. Software update: the ORCA program system, version 4.0. Wiley Interdiscip. Rev.: Comput. Mol. Sci. 2017, 8, e1327.

(43) Neese, F.; Wennmohs, F.; Becker, U.; Riplinger, C. The ORCA quantum chemistry program package. J. Chem. Phys. 2020, 152, 224108. 
(44) Raghavachari, K.; Trucks, G. W.; Pople, J. A.; Head-Gordon, M. A fifthorder perturbation comparison of electron correlation theories. Chem. Phys. Lett. 1989, 15\%, 479-483.

(45) Kendall, R. A.; Dunning, T. H.; Harrison, R. J. Electron affinities of the firstrow atoms revisited. Systematic basis sets and wave functions. J. Chem. Phys. 1992, 96, 6796-6806.

(46) Woon, D. E.; Dunning, T. H. Gaussian basis sets for use in correlated molecular calculations. III. The atoms aluminum through argon. J. Chem. Phys. 1993, 98, 1358-1371.

(47) Prascher, B. P.; Woon, D. E.; Peterson, K. A.; Dunning, T. H.; Wilson, A. K. Gaussian basis sets for use in correlated molecular calculations. VII. Valence, corevalence, and scalar relativistic basis sets for Li, Be, Na, and Mg. Theor. Chem. Acc. 2010, 128, 69-82.

(48) Christiansen, O.; Koch, H.; Jørgensen, P. Response functions in the CC3 iterative triple excitation model. J. Chem. Phys. 1995, 103, 7429-7441.

(49) Koch, H.; Christiansen, O.; Jørgensen, P.; de Merás, A. M. S.; Helgaker, T. The CC3 model: An iterative coupled cluster approach including connected triples. $J$. Chem. Phys. 1997, 106, 1808-1818.

(50) Becke, A. D. Density-functional thermochemistry. III. The role of exact exchange. J. Chem. Phys. 1993, 98, 5648-5652.

(51) Stephens, P. J.; Devlin, F. J.; Chabalowski, C. F.; Frisch, M. J. Ab initio calculation of vibrational absorption and circular dichroism spectra using density functional force fields. J. Phys. Chem. 1994, 98, 11623-11627.

(52) Becke, A. D. A new mixing of Hartree-Fock and local density-functional theories. J. Chem. Phys. 1993, 98, 1372-1377.
(53) Adamo, C.; Barone, V. Toward reliable density functional methods without adjustable parameters: the PBE0 model. J. Chem. Phys. 1999, 110, 6158-6170.

(54) Ernzerhof, M.; Scuseria, G. E. Assessment of the Perdew-Burke-Ernzerhof exchangecorrelation functional. J. Chem. Phys. 1999, 110, 5029-5036.

(55) Chai, J.-D.; Head-Gordon, M. Systematic optimization of long-range corrected hybrid density functionals. J. Chem. Phys. 2008, 128, 084106.

(56) Karton, A.; Tarnopolsky, A.; Lamère, J.F.; Schatz, G. C.; Martin, J. M. L. Highly accurate first-principles benchmark data sets for the parametrization and validation of density functional and other approximate methods. Derivation of a robust, generally applicable, double-hybrid functional for thermochemistry and thermochemical kinetics. J. Phys. Chem. A 2008, 112, 12868-12886.

(57) Brémond, E.; Adamo, C. Seeking for parameter-free double-hybrid functionals: the PBE0-DH model. J. Chem. Phys. 2011, 135, 024106.

(58) Brémond, E.; Pérez-Jiménez, A. J.; Sancho-García, J. C.; Adamo, C. Rangeseparated hybrid density functionals made simple. J. Chem. Phys. 2019, 150, 201102.

(59) Brémond, E.; Sancho-García, J. C.; PérezJiménez, A. J.; Adamo, C. Communication: double-hybrid functionals from adiabatic-connection: The QIDH model. J. Chem. Phys. 2014, 141, 031101.

(60) Brémond, E.; Savarese, M.; PérezJiménez, A. J.; Sancho-García, J. C.; Adamo, C. Range-separated doublehybrid functional from nonempirical constraints. J. Chem. Theory Comput. 2018, 14, 4052-4062.

(61) Sears, J. S.; Koerzdoerfer, T.; Zhang, C.R.; Brédas, J.-L. Communication: orbital 
instabilities and triplet states from timedependent density functional theory and long-range corrected functionals. J. Chem. Phys. 2011, 135, 151103.

(62) Peach, M. J. G.; Tozer, D. J. Overcoming low orbital overlap and triplet instability problems in TDDFT. J. Phys. Chem. A 2012, 116, 9783-9789.

(63) Peach, M. J.; Warner, N.; Tozer, D. J. On the triplet instability in TDDFT. Mol. Phys. 2013, 111, 1271-1274.

(64) Rangel, T.; Hamed, S. M.; Bruneval, F.; Neaton, J. B. An assessment of low-lying excitation energies and triplet instabilities of organic molecules with an ab initio Bethe-Salpeter equation approach and the Tamm-Dancoff approximation. J. Chem. Phys. 2017, 146, 194108.

(65) Grimme, S. In Reviews in computational chemistry; Lipkowitz, K. B., Boyd, D. B., Eds.; Wiley-VCH: New York, 2004; Vol. 20, pp. 153-218.

(66) Jacquemin, D.; Duchemin, I.; Blondel, A.; Blase, X. Benchmark of Bethe-Salpeter for triplet excited-states. J. Chem. Theory Comput. 2017, 13, 767-783.

(67) Goerigk, L.; Grimme, S. A thorough benchmark of density functional methods for general main group thermochemistry, kinetics, and noncovalent interactions. Phys. Chem. Chem. Phys. 2011, 13, 6670-6688.

(68) Goerigk, L.; Hansen, A.; Bauer, C.; Ehrlich, S.; Najibi, A.; Grimme, S. A look at the density functional theory zoo with the advanced GMTKN55 database for general main group thermochemistry, kinetics and noncovalent interactions. Phys. Chem. Chem. Phys. 2017, 19, 3218432215 .

(69) Goerigk, L.; Mehta, N. A trip to the density functional theory zoo: warnings and recommendations for the user. Aust. J. Chem. 2019, 72, 563-573.
(70) Martin, J. M. L.; Santra, G. Empirical double-hybrid density functional theory: A 'third way' in between WFT and DFT. Isr. J. Chem. 2020, 60, 787-804.

(71) Mehta, N.; Casanova-Páez, M.; Goerigk, L. Semi-empirical or non-empirical double-hybrid density functionals: which are more robust? Phys. Chem. Chem. Phys. 2018, 20, 23175-23194.

(72) Najibi, A.; Casanova-Páez, M.; Goerigk, L. Analysis of recent BLYPand PBE-based range-separated doublehybrid density functional approximations for main-group thermochemistry, kinetics, and noncovalent interactions. J. Phys. Chem. A 2021, 125, 4026-4035.

(73) Tozer, D. J.; Handy, N. C. On the determination of excitation energies using density functional theory. Phys. Chem. Chem. Phys. 2000, 2, 2117-2121.

(74) Maitra, N. T.; Zhang, F.; Cave, R. J.; Burke, K. Double excitations within timedependent density functional theory linear response. J. Chem. Phys. 2004, 120, 5932-5937.

(75) Kucharski, R. J., S. A.; Bartlett Recursive intermediate factorization and complete computational linearization of the coupled-cluster single, double, triple, and quadruple excitation equations. Theor. Chim. Acta 1991, 80, 387-405.

(76) Mester, D.; Kállay, M. Accurate spectral properties within double-hybrid density functional theory: a spin-scaled range-separated second-order algebraicdiagrammatic construction-based approach. J. Chem. Theory Comput. 2022, $18,865-882$.

(77) Schirmer, J. Beyond the random-phase approximation: a new approximation scheme for the polarization propagator. Phys. Rev. A 1982, 26, 2395-2416. 
Graphical TOC Entry

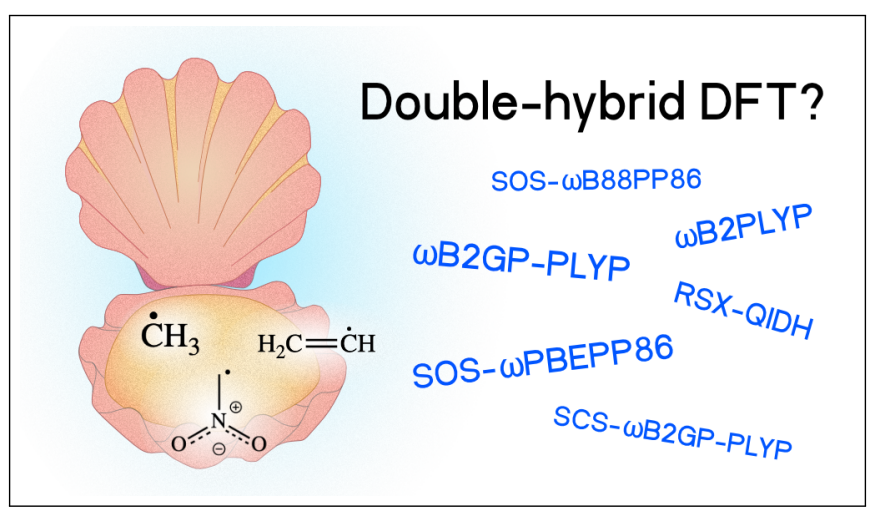

